\title{
Nanobeacon and Laser Beacon: KM3NeT Time Calibration Devices
}

\author{
Diego Real ${ }^{1}$ \\ IFIC, Instituto de Física Corpuscular, Valencia, Spain \\ realeific.uv.es \\ David Calvo \\ IFIC, Instituto de Física Corpuscular, Valencia, Spain \\ dacaldialific.uv.es
}

on behalf of the KM3NeT collaboration

\begin{abstract}
A very large volume neutrino telescope is being constructed in the Mediterranean Sea by the KM3NeT collaboration. Thousands of glass spheres holding a set of 31 small area photomultipliers will be deployed at high depth forming a tri-dimensional matrix. The glass spheres, called Digital Optical Modules (DOMs), will detect the Cerenkov light induced by charged particles produced in neutrino interactions with the surrounding matter. Time synchronization between photomultipliers is required to achieve optimal performances. Two different types of time calibration will be performed in KM3NeT: Intra Detection Unit (DU) calibration, where DOMs of the same DU are synchronized by means of a nanobeacon, an electronics board flashing a LED integrated in DOM, and Inter DU calibration, where synchronization is performed among different Dus, using the Laser Beacon, an instrument installed at the bottom of the detector. Both devices are presented in this contribution.
\end{abstract}

Technology and Instrumentation in Particle Physics 2014

2-6 June, 2014

Amsterdam, the Netherlands

\footnotetext{
${ }^{1}$ Corresponding Author
} 


\section{Introduction}

The KM3NeT telescope is part of a deep sea infrastructure to be deployed at the bottom of the Mediterranean Sea. In Autumn 2014 the construction of the first bunch of 31 Detection Units (DU) of the telescope will start. Their deployment is foreseen in 2015. The main objective is to detect extraterrestrial neutrinos with energies above $100 \mathrm{GeV}$.

The detection principle in a neutrino telescope relies on the measurement of the Cherenkov light emitted in a natural transparent medium, like water, along the path of the charged particles produced in neutrino interactions in the vicinity of a three dimensional array of photon detectors. The arrival time of the light collected by optical detectors, together with position information, is used to reconstruct the muon trajectory, and consequently that of the parent neutrino, which is almost collinear for energy larger than $1 \mathrm{TeV}$. The KM3NeT detector will consist of a tri-dimensional array of digital optical modules, arranged in vertical strings (DUs) that will be moored on the seabed at depths of several kilometers. Each DU will host 18 DOMs distributed over a length of about $700 \mathrm{~m}$. Horizontally, the DUs are separated by about $100 \mathrm{~m}$. DOM made of 31 3" PMTs housed inside a glass sphere resistant to the high pressure present at the sea bottom, the active bases for power and the readout electronics. According to the all-datato shore approach, relevant information are sent to a PC farm on shore after the digitalization. The angular resolution of the reconstructed track depends on the accurate measurement of the arrival time of Cherenkov photons reaching the photo-sensors. Time and position calibration of the detector is therefore of utmost importance to achieve a good angular resolution. For $\mathrm{KM} 3 \mathrm{NeT}$ the required precision of a time calibration system to measure the relative time between photo-sensors should be $\sigma \leq 1 \mathrm{~ns}$. LEDs (Light Emission Diodes) and lasers located throughout the detector produce short duration and powerful light pulses which are detected by the photo-sensors, allowing the measurement of the time delay between the arrival of the photon to the photocathode and the time stamping in the front-end electronics. In KM3NeT the Time Calibration procedure will be performed with two different techniques [1]:

Intra DU Calibration: LED beacons, called Nanobeacon in KM3NeT, will be used to DOMs in the same DU. The system determines the time offset of the DOMs of the same DU. The Nanobeacon is housed in the DOM and its LED will point upwards. In order to have redundancy, the Nanobeacon will be installed in all DOMs.

Inter DU Calibration: Laser Beacons will be used to DOMs of different DUs. The light emitted sidewards by Laser Beacons, located at the bottom of the detector in opportune positions, will illuminate the lowest DOM of several neighboring DUs.

\section{The Laser Beacon}

The KM3NeT Laser Beacon (LB) is a device that emits light by means of a diode pumped Q- switched Nd-YAG laser head. The laser head, together with all the associated electronics to control the device, is housed inside a cylindrical titanium container resistant to high pressure and seawater corrosion. A voltage-controlled optical attenuator, consisting of a liquid crystal retarder and a linear polarizing beam-splitter cube, is used in order to control remotely the light 
intensity emitted by the laser. A glass rod, mounted in an opening in the top-cap of the container, allows the laser beam to go outside. A flat disk diffuser, which spreads the light beam outside following a Lambertian distribution, is mounted in the inner side of the glass rod. Hence the light leaves the cylinder through its vertical wall where biofouling is negligible. Meanwhile The upper part of the glass rod is painted in black to prevent the light from going outside on this side. The dimensions of the glass rod and of the top-cap have been calculated carefully to maximize the amount of light going through the walls of the glass rod. The bottom end-cap of the titanium container holds a penetrator for the power supply and the external communications. Currently two KM3NeT Laser Beacon prototypes have been produced and installed on the Instrumentation Line of ANTARES and on the NEMO Tower Phase II.

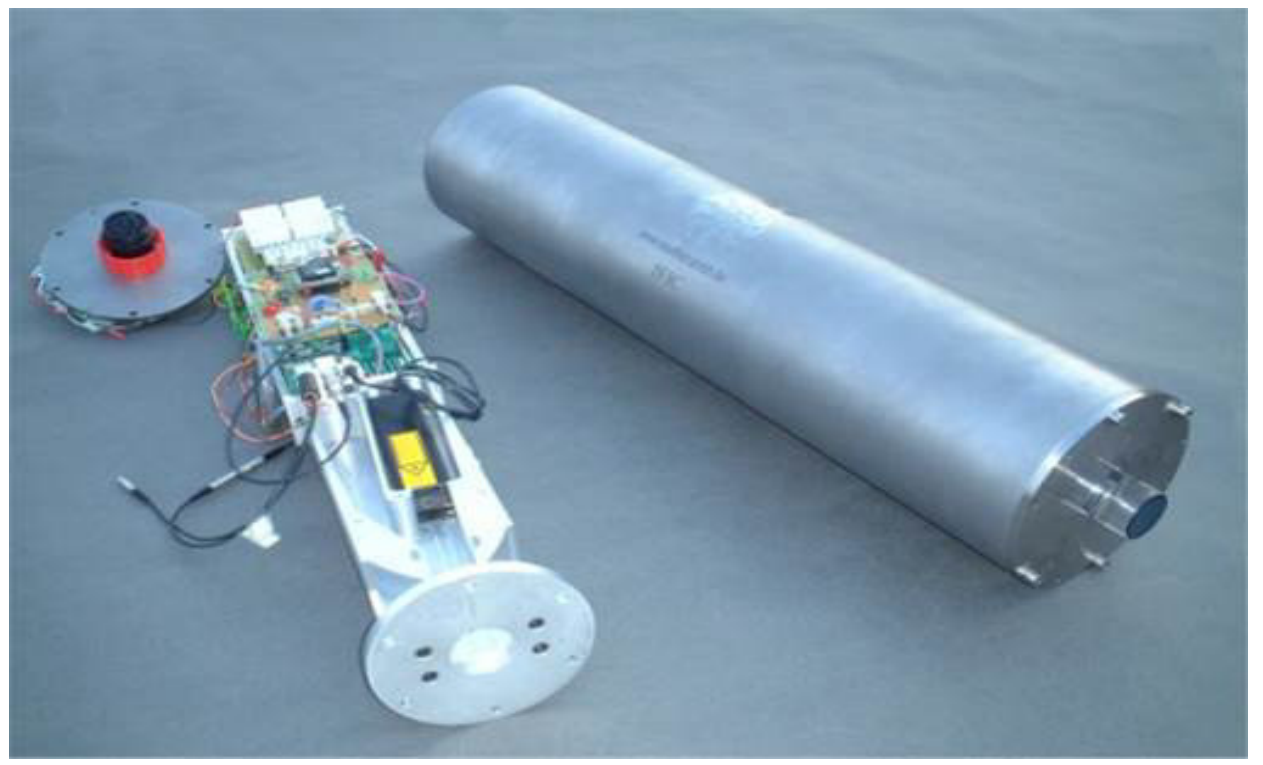

Figure 1: A Laser Beacon open. It is possible to observe the glass rod, with it top painted in black, the penetrator, the inner mechanics, the laser head and all the power and control electronics

\section{The Nanobeacon}

The main aim of the Nanobeacon is to perform intra DU calibration that is between among the DOMs of the same Detection Unit. A Nanobeacon is integrated in the DOM and consists of a small electronics board that controls an LED pointing upwards. The mechanical integration inside the DOM avoids the need of using a mechanical container for the device which and reduces substantially the cost of this calibration device. The LED emits an ultra- short light pulse ( 2-3 ns of rise time) when the appropriate command is received. The main component of the Nanobeacon electronics is the pulser that provides the electrical signal to enable the LED to flash. The Nanobeacon light intensity and rate of emission can be changed. In order to do it the pulser is controlled by means of two control signals controlled remotely via I2C. Geometrical considerations show that a $15^{\circ}$ opening angle is sufficient to illuminate DOMs located on the same D.U. reducing potential misalignments to less smaller than $10^{\circ}$. The total number of devices to be installed in KM3NeT has already been fixed decided and each DOM 
will contain a Nanobeacon. At present 8 Nanobeacon prototypes have been already integrated in the KM3NeT Tower Phase II and 3 more in the PPM KM3NeT DU.

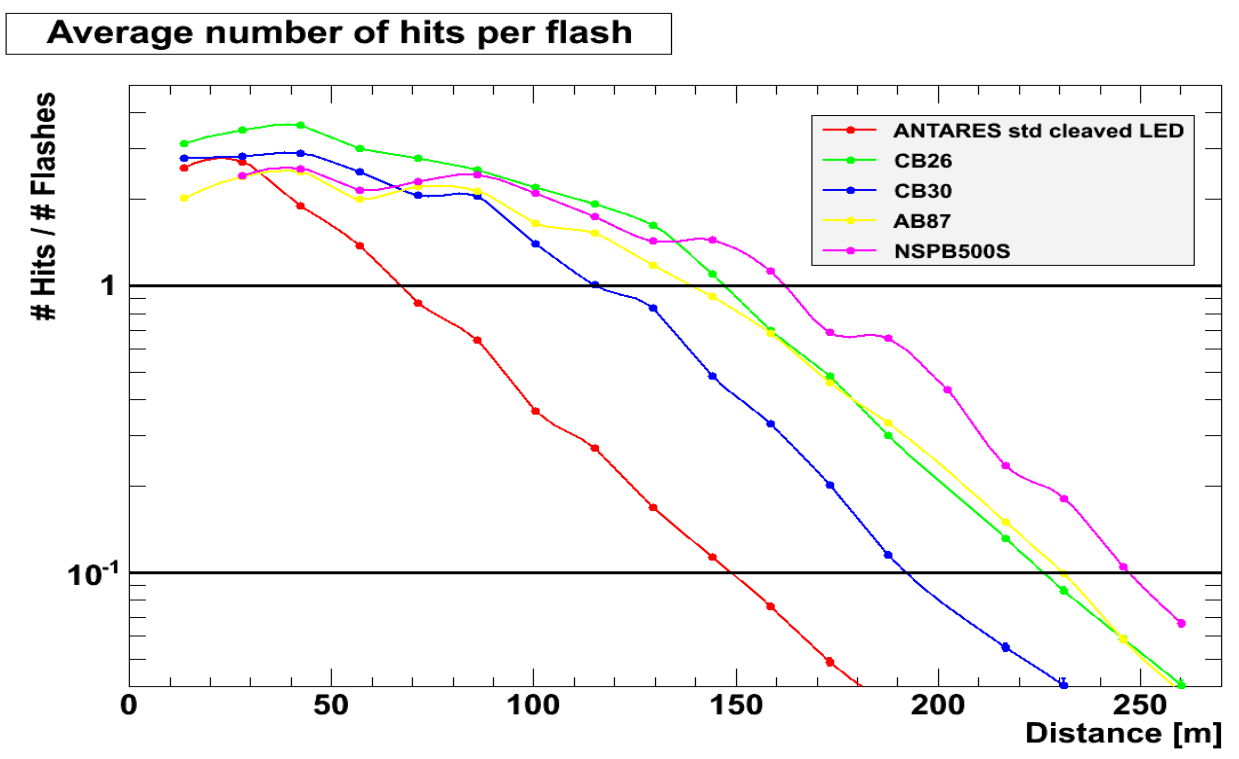

Figure 2. Average number of hits (photo - electrons) per flash as a function of the distance. For the four preselected models measurements were carried out in the ANTARES detector.

\section{Summary}

The laser beacon designed for KM3Net has been presented. Two prototypes have already been successfully deployed: one laser beacon integrated in the ANTARES IL11 (3.5 FJ) and another laser beacon has been integrated in "KM3NeT Nemo Tower Phase II" (3.5 FJ). Currently one more powerful head with 25 FJ per pulse is under evaluation.The Nanobeacon designed for KM3NeT has been presented. 4 precandidates LED has been evaluated in ANTARES. Eight prototypes have already been successfully deployed in the "KM3NeT Nemo Tower Phase II" and one in the PPM DOM. Currently three of them have been integrated in the PPM DU of KM3NeT.

\section{Acknowledgments}

The author acknowledges the financial support of the Spanish Ministerio de Ciencia e Innovacion (MICINN),grants FPA2009-13983-C02-01, FPA2012-37528-C02-01, ACI20091020, Consolider MultiDark CSD2009-00064,RYC-2012-10604 and of the Generalitat Valenciana, Prometeo/2009/026.

\section{References}

1. J.A. Aguilar et al. "Time Calibration of the ANTARES neutrino Telescope" Astroparticle Physics (2011) vol 34. pp 539-549 